\title{
Intra-articular injection of culture-expanded mesenchymal stem cells with or without addition of platelet-rich plasma is effective in decreasing pain and symptoms in knee osteoarthritis: a controlled, double-blind clinical trial
}

\author{
Ricardo Bastos ${ }^{1,2,3,4,5,15}$. Marcelo Mathias ${ }^{2} \cdot$ Renato Andrade $^{1,3,6} \cdot$ Ronaldo J. F. C. Amaral7,8,9 $^{7}$ \\ Vinicius Schott ${ }^{2}$. Alex Balduino ${ }^{10} \cdot$ Raquel Bastos $^{11}$ - J. Miguel Oliveira ${ }^{4,5,12} \cdot$ Rui L. Reis $^{4,5,12} \cdot$ Scott Rodeo $^{13}$. \\ João Espregueira-Mendes $1,3,5,14$ (B)
}

Received: 3 July 2019 / Accepted: 25 September 2019

(c) European Society of Sports Traumatology, Knee Surgery, Arthroscopy (ESSKA) 2019

\begin{abstract}
Purpose To compare the clinical and laboratory outcomes of intra-articular injections of culture-expanded bone-derived mesenchymal stem cells (MSCs) with or without platelet-rich plasma (PRP) to intra-articular corticosteroid injections for the treatment of knee osteoarthritis (OA).

Methods Forty-seven patients with radiographic and symptomatic knee OA were randomized into three groups for intraarticular injections: autologous bone marrow-derived culture-expanded MSCs $(n=16)$; autologous bone marrow-derived culture-expanded MSCs + PRP $(n=14)$; and corticosteroid $(n=17)$. The outcomes were assessed by the Knee Injury and Osteoarthritis Outcome Score (KOOS) and range of motion (ROM) at baseline, 1, 2, 3, 6, 9 and 12 months and intra-articular cytokines analysis at baseline, 6 and 12 months postoperatively.

Results The three groups showed significant improvement in most KOOS domains and global score at 1st month and all domains and global score at 12-month follow-up $(p<0.05)$. At the 1st month, only the MSCs group showed significant differences in KOOS symptoms domain $(p=0.003)$. The MSCs and MSCs + PRP groups showed the highest percentage of improvement in most KOOS domains and global score compared to the corticosteroid group. All three groups showed a significant reduction in intra-articular levels of human interleukin-10 cytokine, from baseline to 12 months $(p<0.05)$.

Conclusion An intra-articular injection of bone marrow-derived culture-expanded MSCs with or without the addiction of PRP is effective in improving the function and decreasing symptoms caused by knee OA at 12-month follow-up.

Level of evidence II.
\end{abstract}

Keywords Osteoarthritis $\cdot$ Mesenchymal stem cells $\cdot$ Intra-articular injection $\cdot$ Platelet-rich plasma

\section{Introduction}

Progressive ageing and a more active population increase the challenge to treat osteoarthritis (OA), which has led to the development of tissue engineering and cell-based therapies. The role of mesenchymal stem cells (MSCs) and

Electronic supplementary material The online version of this article (https://doi.org/10.1007/s00167-019-05732-8) contains supplementary material, which is available to authorized users.

João Espregueira-Mendes

espregueira@dhresearchcentre.com

Extended author information available on the last page of the article platelet-rich plasma (PRP) therapies in knee OA is still controversial [7, 15, 18, 37, 39].

The therapeutic use of MSCs is traditionally related to both their anti-inflammatory activity and the multilineage differentiation, including the chondrogenic potential [22]. Recent studies emphasize the paracrine effects of implanted cells, i.e., the MSCs secretion of cytokines, growth factors and extracellular vesicles capable of inducing tissue repair and modulating inflammation [41]. As MSCs are an extremely rare population within the bone marrow $(0.001-0.01 \%)$ [42], an optimized culture and in vitro expansion standardized protocol could be beneficial for MSCs regenerative success in the clinics [16]. Several growth 
factors involved in MSCs chondrogenic differentiation are found in PRP, such as transforming growth factor beta (TGF- $\beta$ ), basic fibroblast growth factor (bFGF) and platelet-derived growth factor (PDGF) [2]. The PRP, being an autologous source of growth factors, can be easily obtained from peripheral blood but has showed conflicting results for knee OA treatment when compared to hyaluronic acid or corticosteroid intra-articular injections $[7,8,10,12,13,17$, 23, 33, 37].

There is still insufficient evidence to recommend the use of intra-articular injections of MSCs due to the heterogeneity in preparation protocols and high risk of bias [18, 39, 43], but recent systematic reviews reported beneficial effects of stem cell intra-articular injections for knee OA [36, 39]. This randomized, controlled, double-blind clinical trial has the objective to compare the clinical and laboratory outcomes of intra-articular injection of expanded MSCs with or without PRP to corticosteroid intra-articular injection for the treatment of knee OA. The main hypothesis was that cultureexpanded MSCs can lead to better outcomes as compared to corticosteroid treatment. In addition, it was hypothesized that expanded MSCs enhanced with PRP will result in better clinical outcomes as compared to MSCs alone and corticosteroid intra-articular injections.

\section{Materials and methods}

All participants were submitted to an initial screening visit including history taking, physical examination, laboratory testing pre-operative, electrocardiogram, chest radiography, knee radiography (standing anterior-posterior and lateral views), knee magnetic resonance imaging (MRI) and a survey of recently used medications and supplements. All patients were examined by a cardiologist for surgical risk stratification. The inclusion criteria was patients over age 35 with knee OA (based on American College of Rheumatology criteria) and confirmatory radiographs (Kellgren-Lawrence grade 1-4). Exclusion criteria were history of untreated diabetes mellitus, glaucoma, immunodeficiency, chronic use of oral corticosteroid or immunosuppressive therapies, history or presence of malignant disorders and/or use of chemotherapy, infection or active wound in the knee area, history of severe trauma to the knee (post-traumatic OA), presence of systemic inflammation, body mass index (BMI) higher than $40 \mathrm{~kg} / \mathrm{m}^{2}$, pregnancy and any other comorbidity that prevented the bone marrow aspiration surgical procedure.

Initially, a total of 50 patients were assessed for eligibility. Three were excluded before randomization because they did not meet the inclusion criteria (leg infection, erysibelas; $n=1)$ or refused to participate $(n=2)$. A homogenous sample of 47 patients (47 knees; 24 males and 23 females; $57.3 \pm 10.7$ years old) with radiographic and symptomatic knee OA met the inclusion criteria and were included in this study.

As MSCs therapy with cultured expanded cells is an innovative treatment, there are possible undesirable effects. For this reason, the ethical committee authorized to analyse the results of the first patients treated with MSCs therapy who finished their 12-month follow-up aiming to identify possible complications due to the MSCs injections. The safety analysis for the patients that had completed the 12-month follow-up was only performed after the last enrolled patient received the allocated injection. Thus, the randomization and the blinding of patients were not disrupted. This analysis was published in a preliminary paper published (phase 1 study), which comprised a partial sample of patients (18 patients) that are included in this study. The current study shows different results, with the complete sample of patients and including the comparison with the control group and cytokines analyses.

\section{Patient baseline characteristics}

Baseline sociodemographic and clinical characteristics of the three groups are summarized in Table 1. At baseline, the sample was homogeneous regarding gender, age, BMI, knee ROM and KOOS subscales and global scores $(p>0.05)$. One patient deceased at 9 months and was lost to follow-up, and another required total knee replacement at 6 months and was considered as a treatment failure (Fig. 1).

\section{Randomization and blinding procedure}

Patients were randomized just after the bone marrow aspirate and before the scheduled intra-articular injection procedure. Using the Excel software, the included patients were randomly divided into three groups, in a 1:1 ratio using central and permuted-blocks randomization.

1. Autologous transplantation of culture-expanded bone marrow stromal MSCs intra-articular injection;

2. Autologous transplantation of expanded stromal bone marrow MSCs combined with PRP intra-articular injection;

3. Corticosteroid intra-articular injection.

The biologist who prepared the material to be injected had restricted access to the randomization schedule, which specified the allocated treatment group. The orthopaedic surgeon, patients, and staff directly involved in the study were blinded to group assignment. Only the biologist involved with the preparation of the material to be injected knew the patient allocation. During treatment preparation, the therapeutic solutions were packed in a standard syringe and covered with a blank protector to ensure blinding. Solutions 
Table 1 Baseline sociodemographic and clinical characteristics

\begin{tabular}{|c|c|c|c|c|}
\hline & $\operatorname{MSCs}(n=16)$ & $\mathrm{MSCs}+\mathrm{PRP}(n=14)$ & Corticosteroid $(n=17)$ & $p$ value \\
\hline \multicolumn{5}{|l|}{ Gender } \\
\hline Female & $6(37.5 \%)$ & $9(64.3 \%)$ & $8(47.1 \%)$ & \multirow[t]{2}{*}{ n.s. ${ }^{*}$} \\
\hline Male & $10(62.5 \%)$ & $5(35.7 \%)$ & $9(52.9 \%)$ & \\
\hline Age, mean \pm SD & $55.7 \pm 7.8$ & $60.8 \pm 9.9$ & $55.9 \pm 13.4$ & n.s. ${ }^{* *}$ \\
\hline $\mathrm{BMI}$, mean $\pm \mathrm{SD}$ & $30.6 \pm 4.5$ & $28.9 \pm 4.9$ & $31.0 \pm 4.7$ & n.s.** \\
\hline$<30 \mathrm{~kg} / \mathrm{m}^{2}$ & $43 \%$ & $64 \%$ & $47 \%$ & \\
\hline$\geq 30 \mathrm{~kg} / \mathrm{m}^{2}$ & $57 \%$ & $36 \%$ & $53 \%$ & \\
\hline \multicolumn{5}{|l|}{ Alignment, no. (\%) } \\
\hline Normal alignment & $3(18.8 \%)$ & $6(42.9 \%)$ & $7(41.2 \%)$ & \multirow[t]{3}{*}{ n.s. ${ }^{*}$} \\
\hline Varus & $13(81.3 \%)$ & $5(35.7 \%)$ & $4(23.5 \%)$ & \\
\hline Valgus & $0(0.0 \%)$ & $3(21.4 \%)$ & $6(35.3 \%)$ & \\
\hline \multicolumn{5}{|l|}{ OA grade, no. $(\%)$} \\
\hline Grade I & $1(6.3 \%)$ & $1(7.1 \%)$ & $1(5.8 \%)$ & \\
\hline Grade II & $7(43.8 \%)$ & $3(21.4 \%)$ & $4(23.5 \%)$ & \\
\hline Grade III & $5(31.3 \%)$ & $6(42.9 \%)$ & $7(41.1 \%)$ & \\
\hline Grade IV & $3(18.8 \%)$ & $4(28.6 \%)$ & $5(29.4 \%)$ & \\
\hline \multicolumn{5}{|c|}{$\mathrm{ROM}$, mean $\pm \mathrm{SD}(95 \% \mathrm{CI})$} \\
\hline Hyperextension & $2.2 \pm 4.1(0.0,4.4)$ & $0.7 \pm 2.7(-0.8,2.3)$ & $1.0 \pm 2.1(-0.1,2.1)$ & n.s. $* * *$ \\
\hline Flexum & $2.5 \pm 5.8(-0.6,5.6)$ & $3.2 \pm 4.6(0.5,5.9)$ & $3.9 \pm 6.3(0.4,7.3)$ & n.s. $* * *$ \\
\hline Total flexion & $104.4 \pm 39.4(83.4,125.4)$ & $115.2 \pm 26.6(99.9,130.6)$ & $118.3 \pm 18.3(108.2,128.5)$ & n.s. $* * *$ \\
\hline \multicolumn{5}{|c|}{ KOOS, mean $\pm \mathrm{SD}(95 \% \mathrm{CI})$} \\
\hline Symptoms & $41.5 \pm 18.4(31.7,51.3)$ & $44.6 \pm 14.9(36.1,53.2)$ & $47.4 \pm 17.9(38.2,56.6)$ & n.s. ${ }^{* *}$ \\
\hline Pain & $34.5 \pm 11.4(28.5,40.6)$ & $42.3 \pm 17.2(32.3,52.2)$ & $40.5 \pm 19.6(30.4,50.6)$ & n.s. ${ }^{* *}$ \\
\hline Function, daily living & $31.7 \pm 19.1(21.5,41.9)$ & $41.2 \pm 18.2(30.7,51.7)$ & $40.7 \pm 21.0(29.9,51.4)$ & n.s. $* *$ \\
\hline Sports/recreation & $13.1 \pm 21.0(2.0,24.3)$ & $18.9 \pm 23.5(5.4,32.5)$ & $18.2 \pm 28.3(3.7,32.8)$ & n.s. $* * *$ \\
\hline Quality of life & $16.8 \pm 12.4(10.1,23.4)$ & $19.2 \pm 20.3(7.5,30.9)$ & $16.5 \pm 16.5(8.0,25.0)$ & n.s. $* *$ \\
\hline Global KOOS score & $30.3 \pm 13.1(23.3,37.3)$ & $37.3 \pm 16.4(27.8,46.7)$ & $36.9 \pm 17.8(27.7,46.0)$ & n.s. $* *$ \\
\hline
\end{tabular}

$S D$ standard deviation, $C I$ confidence interval, $O A$ osteoarthritis, ROM range of movement, KOOS Knee Injury and Osteoarthritis Outcome Score, n.s. non-significant

*Qui square test; **ANOVA; ***Kruskall-Wallis

were prepared to the same final volume and weight to prevent identification of the different treatments.

\section{MSC harvest, cell culturing and syringe preparation}

All patients were submitted to bone marrow aspiration procedure independently of the group allocated, including the corticosteroid group. Bone marrow aspirate was obtained percutaneously from both posterior iliac crests with a Jamshidi needle $(11 \mathrm{G} \times 10 \mathrm{~cm}$; Ecomed, Rio de Janeiro, Brazil) after spinal nerve block and sedation. Approximately 80-100 mL of bone marrow was aspirated through $10 \mathrm{~mL}$ EDTA-coated syringes (ten syringes used on the left posterior iliac crest side and ten syringes used on the right side). Each syringe was filled with 4-5 mL of bone marrow aspiration and the Jamshidi needle was introduced to different iliac crest sites to prevent the aspiration of venous blood. The syringes were previously rinsed with anticoagulant
(EDTA). Mononuclear cells from bone marrow samples were separated using the Sepax automated closed system (Biosafe, Eysins, Switzerland), according to manufacturer's instructions. After isolation, cells were seeded at a density of $4 \times 10^{5}$ cells $/ \mathrm{cm}^{2}$ in minimum essential medium eagle alpha modification (alpha MEM) (Cultilab, Campinas, SP, Brazil) supplemented with $10 \%$ foetal bovine serum (FBS) (Gibco) and incubated for 5 days in a humidified atmosphere of $5 \% \mathrm{CO}_{2}$ at $37^{\circ} \mathrm{C}$. Non-adherent cells were then discarded after cell culture medium exchange. Medium exchange was performed every 3-4 days. Once the cells reached 70-80\% confluence, they were detached from culture flasks using $0.05 \%$ trypsin solution (Gibco, Thermo Fisher Scientific) and re-seeded onto new culture flasks (Corning Incorporated, Corning, NY), characterizing one passage. Cultures were maintained for no more than two passages. The cells met the criteria for mesenchymal stromal cells (MSC) based on colony-forming unit-fibroblast (CFU-F) assay and flow 


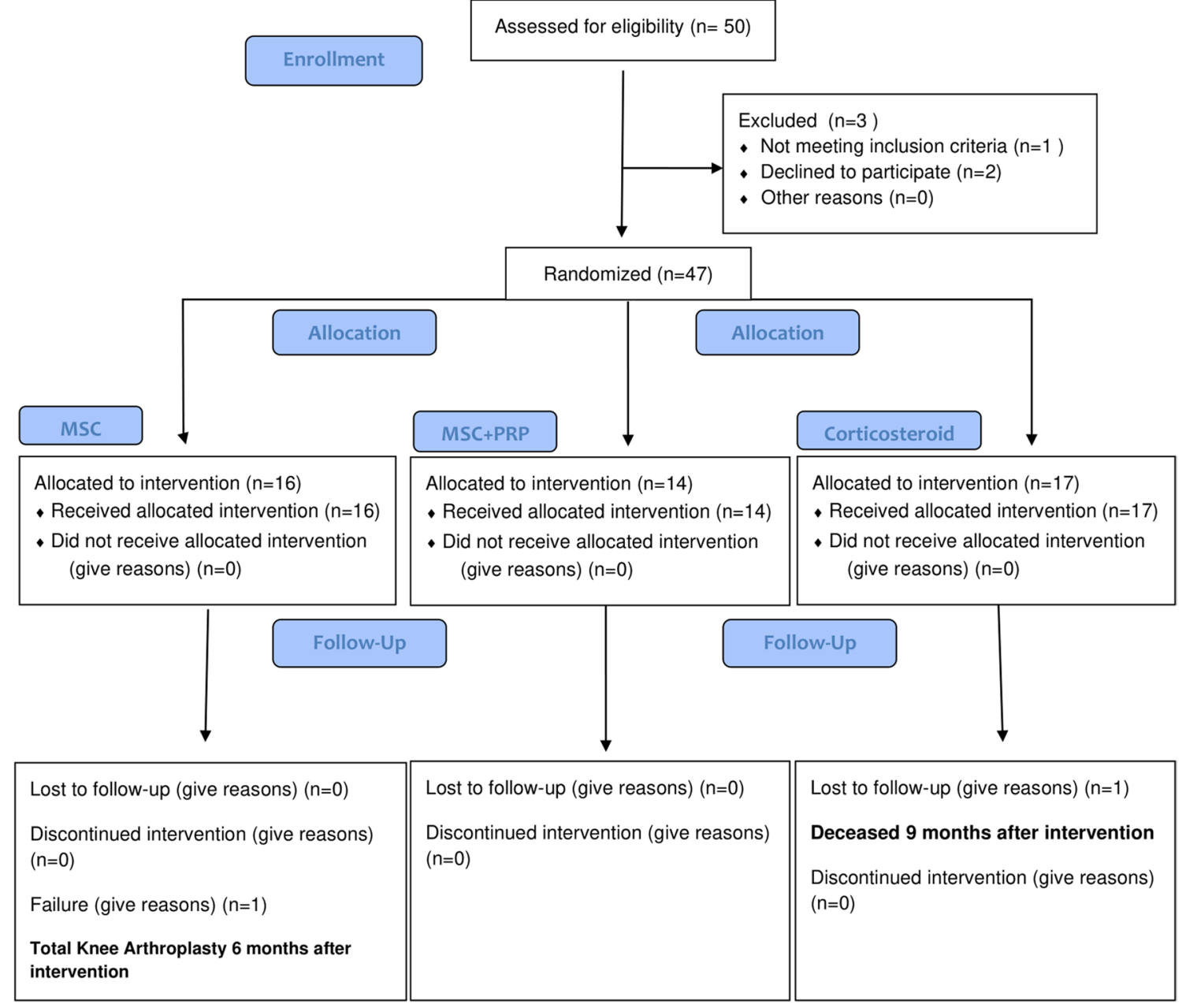

Fig. 1 Consort flow chart of participants

cytometry as previously described [5]. When reaching the desired number of $40 \times 10^{6}$, cells were suspended in $10 \mathrm{~mL}$ PBS supplemented with 2\% human albumin in the MSC group or in PRP in the MSC + PRP group.

\section{PRP preparation}

White blood cell-depleted PRP was produced as previously described, with minor modifications [2]. Only autologous PRP was used. Peripheral blood (54 mL) was collected by venipuncture from each donor using six blood collection tubes containing anticoagulant citrate dextrose (ACD) solution A (Vacutainer, Ref: 364606; BD Biosciences). Under sterile conditions, the blood collected in one ACD tube was divided into three polypropylene tubes containing no anticoagulant (FalconTM, Ref: 352063; BD Biosciences), to enhance platelet recovery. After two centrifugation steps, platelets were re-suspended in phosphate buffer solution (PBS) at a concentration of $10^{6} / \mu \mathrm{L}$, namely PRP, of which
$10 \mathrm{~mL}$ was used to resuspend MSCs in the MSC + PRP group.

\section{Intra-articular injection}

Two to three weeks after the bone marrow aspiration, an intra-articular injection of MSCs, MSCs enriched with PRP, or corticosteroid (4 mg of dexamethasone) was given. Specific intra-articular injection procedures have been previously described [5].

\section{Post-operative and post-injection care}

Neither functional restrictions nor any special care was recommended or required following the bone marrow aspiration. Immediately after injection, patients were instructed to initiate daily prophylactic exercises for preventing deep venous thrombosis, following the same protocol in all study groups. All patients were instructed to remain non-weight bearing with two Canadian-type crutches for 2 weeks to 
favour cell adhesion to chondral lesions. Patients were generally recommended to continue their mild to moderate level of activities and to increase their activity level gradually as tolerated. All activities including moderate sports were allowed after 2 weeks. During the 12 months of follow-up, other therapies such as anti-inflammatory medications (steroid or non-steroidal), physical therapy, or acupuncture were not allowed. The use of dipyrone $1 \mathrm{~g}$ every $6 \mathrm{~h}$ (analgesic non-anti-inflammatory) was allowed in case of severe pain.

\section{Outcome measures}

Medical visits occurred before the bone marrow aspiration procedure (screening visit) and at 1, 2, 3, 6, 9 and 12 months after the experimental treatment by the orthopaedic team.

A clinical and functional assessment, as performed by Portuguese version of the Knee Injury and Osteoarthritis Outcome Score (KOOS) [14], was the primary outcome and was assessed at 1, 2, 3, 6, 9 and 12 months. Using the KOOS subscales and global score, the expected improvement $(\mathrm{IE}=100-$ initial score), the improvement observed $(\mathrm{IO}=$ final score - initial score $)$ and the percentage achieved of the improvement expected ( $\mathrm{CIE}=\mathrm{IO} / \mathrm{IE})$ from baseline to 12-month follow-up was calculated for each patient.

Knee range of motion (ROM) was measured (total active flexion) using Goniômetro Pro for iPhone software and compared to the contralateral knee. Synovial fluid aspiration for cytokine analysis was performed via a superolateral approach at baseline, 6 and 12 months after treatment. Cytokines IL-17A, IFN-gamma, human-TNF, human-IL10, human-IL6, human-IL4, and human-IL2 were analysed when an effusion was present and synovial fluid aspiration was possible. In cases of absence of synovial fluid, the result was considered negative.

\section{Ethical approval}

The research was approved by the Comissão Nacional de Ética em Pesquisa (Brazilian National Ethics in Research Committee)-CONEP, under number 14878813.4.0000.5533 and was conducted in accordance with the principles of the Declaration of Helsinki. All the participants signed the written consent form before inclusion in the study.

\section{Statistical analysis}

The statistical analysis was performed using the Statistical Package for Social Science version $22^{\circledR}$ (IBM SPSS, Inc., Chicago). The level of significance for all hypothesis tests $(p)$ was set at 0.05 . Continuous variables were presented as mean and standard deviation. Absolute $(n)$ and relative $(\%)$ frequencies were computed for the categorical variables. The normality of the distribution of quantitative variables was evaluated by Kolmogorov-Smirnov's test and Shapiro-Wilk's test, but due to the small sample size, the non-parametric tests were performed in all evaluations. The association between baseline categorical and continuous variables was determined using Chi square and ANOVA or Kruskall-Wallis tests, respectively. The comparison of knee ROM and KOOS global and sub-scores during follow-up endpoints between the three groups was made with Kruskall-Wallis test. The Wilcoxon signed-rank test was used to compute the improvement of KOOS global and subscores between each endpoint for each treatment group and the Friedman test to calculate the improvement throughout the entire follow-up. The KOOS improvement from baseline to 12-month follow-up endpoint with the $95 \%$ confidence intervals (CIs) was computed using Wilcoxon signed-rank test. For the cytokine analysis, the Wilcoxon signed-rank test was used to compare the baseline values with the 6-month and 12-month follow-up periods, and the Kruskall-Wallis test to compare the three groups.

\section{Results}

\section{Clinical and functional outcome measures}

\section{KOOS score}

Accounting for all interventions, there was a significant improvement from baseline to 1 month in most KOOS domains and global score $(p<0.05)$ and from baseline to last follow-up for most KOOS domains $(p<0.05$; Supplement 1). The results for each treatment from baseline to 12-month follow-up period are depicted in Table 2 and the changes between all assessments are presented in Supplement 1.

The corticosteroid group had highest percentages for score worsening for KOOS domains and global score and, in turn, the MSCs and MSCs + PRP groups showed the highest percentages of improvement in KOOS domains and global score (Supplement 2).

The MSCs and MSCs + PRP groups achieved higher percentages of expected improvement as compared to the corticosteroid group for most KOOS domains and global score (Fig. 2). When comparing both MSCs groups, the MSCs + PRP group showed higher percentage of expected improvement for the KOOS pain domain, and the MSCs group for the KOOS-QoL domain.

\section{Knee ROM}

There were no significant differences between groups in knee ROM (hyperextension, flexion, and total flexion) at the different follow-up endpoints. 
Table 2 KOOS improvement from baseline to 12-month follow-up

\begin{tabular}{|c|c|c|c|c|}
\hline KOOS & Baseline, mean \pm SD & 12 months, mean \pm SD & Mean difference $(95 \% \mathrm{CI})$ & $p$ value* \\
\hline \multicolumn{5}{|l|}{ Corticosteroid } \\
\hline Symptoms & $47.4 \pm 17.9$ & $56.1 \pm 22.3$ & $8.7(-4.9-22.3)$ & n.s. \\
\hline Pain & $40.5 \pm 19.6$ & $59.5 \pm 22.2$ & $19.0(4.9-33.1)$ & 0.029 \\
\hline Function, daily living & $40.7 \pm 21.0$ & $61.6 \pm 24.4$ & $20.9(5.6-36.2)$ & $\mathbf{0 . 0 3 0}$ \\
\hline Sports/recreation & $18.0 \pm 28.0$ & $36.2 \pm 31.7$ & $17.9(-2.2-38.0)$ & n.s. \\
\hline Quality of life & $16.5 \pm 16.5$ & $32.0 \pm 29.3$ & $15.4(-0.6-31.4)$ & n.s. \\
\hline Global KOOS score & $36.9 \pm 17.8$ & $54.4 \pm 22.7$ & $17.5(3.8-31.2)$ & n.s. \\
\hline \multicolumn{5}{|l|}{ MSCs } \\
\hline Symptoms & $41.5 \pm 18.4$ & $61.6 \pm 22.5$ & $20.1(5.9-34.3)$ & 0.010 \\
\hline Pain & $34.6 \pm 11.4$ & $56.8 \pm 26.5$ & $22.2(8.1-36.3)$ & 0.008 \\
\hline Function, daily living & $31.7 \pm 19.1$ & $58.4 \pm 27.5$ & $26.7(10.3-43.1)$ & 0.003 \\
\hline Sports/recreation & $13.0 \pm 21.0$ & $36.6 \pm 29.5$ & $23.4(5.7-41.1)$ & 0.001 \\
\hline Quality of life & $16.8 \pm 12.4$ & $40.2 \pm 25.9$ & $23.5(9.4-37.6)$ & 0.009 \\
\hline Global KOOS score & $30.3 \pm 13.1$ & $54.2 \pm 24.7$ & $24.0(10.3-37.7)$ & 0.002 \\
\hline \multicolumn{5}{|l|}{$\mathrm{MSCs}+\mathrm{PRP}$} \\
\hline Symptoms & $44.6 \pm 14.9$ & $60.5 \pm 17.6$ & $15.8(3.7-27.9)$ & 0.025 \\
\hline Pain & $42.3 \pm 17.2$ & $65.5 \pm 26.3$ & $23.2(6.7-39.7)$ & $<0.001$ \\
\hline Function, daily living & $41.2 \pm 18.2$ & $66.3 \pm 27.4$ & $25.1(7.9-42.3)$ & 0.004 \\
\hline Sports/recreation & $19.0 \pm 23.0$ & $47.1 \pm 34.5$ & $28.2(6.5-49.9)$ & 0.026 \\
\hline Quality of life & $19.2 \pm 20.3$ & $35.7 \pm 25.6$ & $16.5(-0.6-33.6)$ & n.s. \\
\hline Global KOOS score & $37.3 \pm 16.4$ & $59.9 \pm 24.8$ & $22.7(7.1-38.3)$ & 0.005 \\
\hline
\end{tabular}

Bold values indicate significant $p$ value $(p<0.05)$

$S D$ standard deviation, $C I$ confidence interval, KOOS Knee Injury and Osteoarthritis Outcome Score, n.s. non-significant

*Wilcoxon signed-rank test

Fig. 2 Mean percentage achieved of expected improvement at the 12 months follow-up

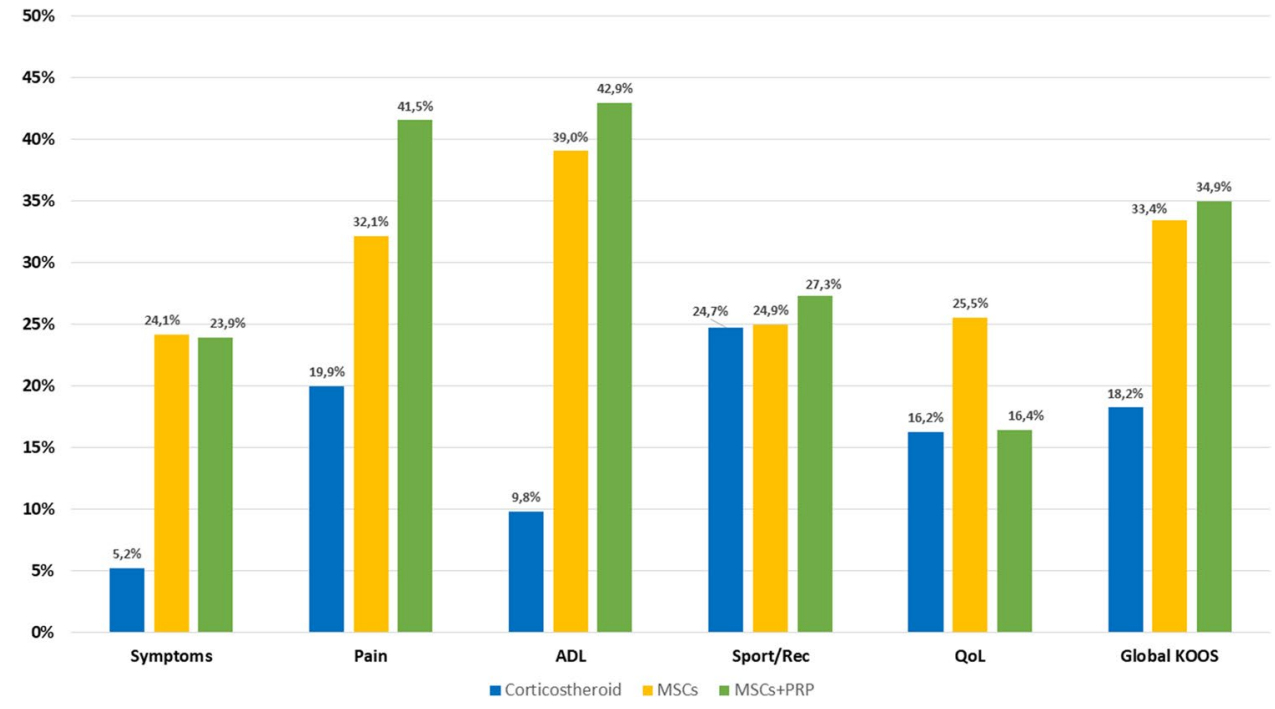

\section{Cytokine analysis}

At baseline, one patient from the MSCs + PRP group did not present synovial fluid to proceed with the cytokine analysis. Eight patients did not present synovial fluid to allow the cytokine analysis at 6 months $(n=3$, MSCs group; $n=1$, MSCs + PRP group; $n=4$, corticosteroid group;) and 11 patients at 12 months ( $n=3$, MSCs group; $n=5$ MSCs + PRP group; $n=3$, corticosteroid group). Thus, these patients were excluded for the final cytokine analysis.

All three groups showed a significant reduction in levels of human-IL10 from baseline to 12 months $(p<0.05)$. The 
corticosteroid group also showed a significant reduction in IL-17A at 12 months $(p=0.033)$. All other studied cytokines did not show significant differences between baseline and 6- and 12-month follow-up endpoints (n.s.; Table 3).

\section{Discussion}

The main finding of this study was that intra-articular injection of expanded MSCs with or without the addition of PRP resulted in a significant improvement in most of KOOS domains and global score after a 12-month follow-up.

Corticosteroid injections have been used for conservative treatment of OA but show short-term efficacy and several local and systemic undesirable effects have been reported
$[3,24,35]$. Despite the similarity of the three groups of treatment during the 1st and 2nd months of treatment, the corticosteroid group show an inferior number of improved KOOS domains from baseline to 12 months and the highest percentage of score worsening for all KOOS domains and global score, excepting KOOS-QoL domain. This finding reinforces the known short-term effect of corticosteroid intra-articular injections. All groups surpassed the minimal clinically important difference for all the KOOS subscales $[11,38]$, but the MSCs and MSCs + PRP groups demonstrate the highest percentage of improvement at 12 months in all KOOS domains and global score, with the exception of MSCs + PRP group in the KOOS -sports-rec and KOOSQoL domains. Pain, symptoms and function subscales are the primary reasons for patients with knee OA seeking

Table 3 Cytokine analysis at baseline and 6- and 12-month follow-up

\begin{tabular}{|c|c|c|c|c|c|c|}
\hline Cytokine & Treatment group & Baseline mean $\pm \mathrm{SD}$ & $\begin{array}{l}\text { 6-month follow-up } \\
\text { mean } \pm S D\end{array}$ & $p^{*}$ & $\begin{array}{l}\text { 12-month follow-up } \\
\text { mean } \pm \mathrm{SD}\end{array}$ & $p^{* *}$ \\
\hline \multirow[t]{4}{*}{ IL-17A, ng/mL } & $\operatorname{MSCs}(n=12)$ & $54.9 \pm 38.4$ & $70.6 \pm 38.3$ & n.s. & $71.6 \pm 38.9$ & n.s. \\
\hline & MSCs + PRP $(n=10)$ & $65.7 \pm 53.0$ & $93.7 \pm 59.4$ & n.s. & $75.1 \pm 37.9$ & n.s. \\
\hline & Corticosteroid $(n=13)$ & $78.9 \pm 54.9$ & $89.2 \pm 63.6$ & n.s. & $41.6 \pm 34.8$ & $\mathbf{0 . 0 3 3}$ \\
\hline & Intergroup sig*** & n.s. & n.s. & & n.s. & \\
\hline \multirow[t]{4}{*}{ IFN-GAMA, ng/mL } & $\operatorname{MSCs}(n=12)$ & $0.06 \pm 0.12$ & $0.26 \pm 0.40$ & n.s. & $0.05 \pm 0.16$ & n.s. \\
\hline & MSCs + PRP $(n=10)$ & $0.95 \pm 1.81$ & $0.54 \pm 1.11$ & n.s. & $0.17 \pm 0.54$ & n.s. \\
\hline & Corticosteroid $(n=13)$ & $0.16 \pm 0.20$ & $0.36 \pm 0.59$ & n.s. & $0.14 \pm 0.34$ & n.s. \\
\hline & Intergroup sig*** & n.s. & n.s. & & n.s. & \\
\hline \multirow[t]{4}{*}{ Human-TNF, ng/mL } & $\operatorname{MSCs}(n=12)$ & $0.00 \pm 0.01$ & $0.32 \pm 0.75$ & n.s. & $0.07 \pm 0.25$ & n.s. \\
\hline & MSCs + PRP $(n=10)$ & $2.23 \pm 4.00$ & $1.11 \pm 2.28$ & n.s. & $0.41 \pm 1.00$ & n.s. \\
\hline & Corticosteroid $(n=13)$ & $0.26 \pm 0.35$ & $0.43 \pm 1.07$ & n.s. & $0.30 \pm 0.74$ & n.s. \\
\hline & Intergroup sig*** & 0.004 & n.s. & & n.s. & \\
\hline \multirow[t]{4}{*}{ Human-IL10, ng/mL } & $\operatorname{MSCs}(n=12)$ & $1.25 \pm 1.17$ & $0.66 \pm 0.90$ & n.s. & $0.26 \pm 0.51$ & 0.023 \\
\hline & MSCs + PRP $(n=10)$ & $3.33 \pm 3.28$ & $1.99 \pm 2.99$ & n.s. & $0.52 \pm 0.99$ & 0.013 \\
\hline & Corticosteroid $(n=13)$ & $1.32 \pm 0.68$ & $1.24 \pm 1.37$ & n.s. & $0.64 \pm 1.32$ & 0.041 \\
\hline & Intergroup sig*** & n.s. & n.s. & & n.s. & \\
\hline \multirow[t]{4}{*}{ Human-IL6, ng/mL } & $\operatorname{MSCs}(n=12)$ & $183.6 \pm 243.9$ & $194.5 \pm 276.5$ & n.s. & $110.6 \pm 249.4$ & n.s. \\
\hline & $\mathrm{MSCs}+\operatorname{PRP}(n=10)$ & $525.4 \pm 1064.5$ & $524.3 \pm 819.4$ & n.s. & $121.1 \pm 107.3$ & n.s. \\
\hline & Corticosteroid $(n=13)$ & $82.8 \pm 89.4$ & $123.7 \pm 164.3$ & n.s. & $80.7 \pm 82.2$ & n.s. \\
\hline & Intergroup sig*** & n.s. & n.s. & & n.s. & \\
\hline \multirow[t]{4}{*}{ Human-IL4, ng/mL } & $\operatorname{MSCs}(n=12)$ & $0.09 \pm 0.22$ & $0.20 \pm 0.40$ & n.s. & $0.04 \pm 0.11$ & n.s. \\
\hline & MSCs + PRP $(n=10)$ & $1.15 \pm 2.12$ & $0.82 \pm 1.61$ & n.s. & $0.19 \pm 0.41$ & n.s. \\
\hline & Corticosteroid $(n=13)$ & $0.22 \pm 0.45$ & $0.37 \pm 0.91$ & n.s. & $0.17 \pm 0.42$ & n.s. \\
\hline & Intergroup sig*** & n.s. & n.s. & & n.s. & \\
\hline \multirow[t]{4}{*}{ Human-IL2, ng/mL } & $\operatorname{MSCs}(n=12)$ & $0.21 \pm 0.34$ & $0.35 \pm 0.62$ & n.s. & $0.08 \pm 0.29$ & n.s. \\
\hline & $\mathrm{MSCs}+\operatorname{PRP}(n=10)$ & $1.38 \pm 3.13$ & $1.45 \pm 2.68$ & n.s. & $0.30 \pm 0.77$ & n.s. \\
\hline & Corticosteroid $(n=13)$ & $0.31 \pm 0.58$ & $0.32 \pm 0.71$ & n.s. & $0.19 \pm 0.48$ & n.s. \\
\hline & Intergroup sig*** & n.s. & n.s. & & n.s. & \\
\hline
\end{tabular}

Bold values indicate significant $p$ value $(p<0.05)$

n.s. non-significant

*Wilcoxon's test comparing baseline and 6-month follow-up measurements; **Wilcoxon's test comparing baseline and 12-month follow-up measurements; ***Kruskall-Wallis test 
medical treatment and thus this superiority is clinically relevant.

Several studies evaluate the benefits of MSCs therapy in association with other surgical procedures such as arthroscopic debridement [29] and osteotomies [28, 30] with or without concomitant microfracture [48]. Controlled clinical trials are scarce and the real influence of the use of biological injections is poorly established $[18,39]$. This study aimed to evaluate the effect of the MSCs therapy alone, without any confounding surgical procedures, anti-inflammatory medication or physiotherapy protocol associated with treatment of OA. Anti-inflammatory medication was not allowed due to its potential effect on the influence of MSCs and PRP in joint inflammation $[6,26,40]$. The exercise regimen followed by all patients consisted of simple self-executed exercises for prevention of deep venous thrombosis and the only active treatment intervention was the intra-articular injection.

Isolated closed cell separation systems are insufficient to obtain the desired therapeutic cell concentration. Laboratory-based cell sorting and in vitro culture expansion are crucial for MSCs regenerative success [9]. During the laboratory phase of the study, MSCs obtained from bone marrow were isolated and expanded until a concentration of the desired number of $4.0 \times 10^{7}$ cells. A recent systematic review of MSC preparation protocols and composition reported a mean number of $8.7 \times 10^{7}$ (range, $8.5 \times 10^{6}$ to $10 \times 10^{8}$ ) cells after culture of bone marrow-derived cells [43]. Previous studies suggest that a higher dose of cells could be important to achieve efficacy and better clinical outcomes [21, 31]. Conversely, scar tissue formation, different from cartilage, following treatment with doses as low as $1.0 \times 10^{7}$ cells was reported in preclinical setting [1]. In this study, a final number of $4.0 \times 10^{7}$ cells were pre-determined. Prolonged in vitro expansion may lead to MSC genomic instability and phenotypic changes. Of note, an early passage MSCs (no more than two passages) was used to assure safety for clinical applications [27, 45].

Few studies were developed to evaluate the effect of the combination of MSCs and PRP on the treatment of osteoarticular conditions, and the interaction between MSCs and PRP is yet not well understood. Studies show conflicting evidence on intra-articular injection of isolated PRP for the treatment of OA [7, 8, 10, 12, 13, 17, 23, 32, 33, 37]. The likely mechanism for a positive effect of PRP on symptom reduction is the production of anti-inflammatory mediators [44]. Another potential mechanism for PRP effect is the stimulation of endogenous progenitor cells, based on the intra-articular delivery of a large pool of growth factors and proteins stored in the alpha-granules of platelets. Several growth factors that participate in MSCs chondrogenic differentiation are in PRP, such as TGF- $\beta$, basic fibroblast growth factor bFGF and PDGF [2], and the addition of PRP to MSCs may improve the reparative properties of the cells.
This study showed similar results between MSC alone and MSCs + PRP groups in terms of clinical outcomes.

Several cytokines have been associated with OA pathophysiology [25]. This study evidenced changes particularly in IL-17A, TNFa and IL-10. Levels of IL-17 are significantly higher in OA patients [34]. These are correlated with increased pain [4] and its injection can induce OA in rabbit knees [46]. Corticosteroid treatment significantly reduced IL-17A levels, but this cytokine was not affected in MSC or MSC + PRP treatment. There was also a decrease in IL-10 levels in all groups. IL-10 is commonly associated with a chondroprotective and anti-inflammatory role in OA [47]. Positive effects of exercise in patients with knee OA were correlated with increase in IL-10 levels. There is also evidence that serum levels of IL-10 is higher in OA patients than in healthy patients and could be associated with pain and function scores [20]. The IL-10 mRNA expression was also shown to be higher in osteoarthritic than in normal chondrocytes [19]. It is possible that high levels of IL-10 are normally present in $\mathrm{OA}$ as a compensatory attempt to decrease the inflammatory environment, and that the decrease in IL-10 levels here observed might be interpreted as a tendency towards the resolution of the inflammation. Although some cytokines showed relevant changes (IL $17 \mathrm{~A}$ and $\mathrm{TNFa}$ ), the small sample size and heterogeneity in cytokine levels among donors may be a reason for the lack of statistical significance (risk of type II error).

The results of this study are of clinical relevance as they show a significant functional and symptomatic benefit in the use of MSCs and MSCs + PRP in patients with symptomatic knee OA. The improvement in most KOOS domains and global score during 12-month follow-up is likely explained by the anti-inflammatory action of MSCs and PRP, ameliorating symptoms caused by synovitis. However, there is no data to suggest that one injection of culture-expanded MSCs with or without PRP is sufficient to promote cartilage growth to repair the chondral damage caused by OA. Further research with high-powered and high-quality randomized controlled trials is warranted to establish the effectiveness of intra-articular injections of MSCs and MSCs with PRP for knee osteoarthritis.

This study has several limitations. A placebo group was not included and the potential of placebo effect in any of the included groups could not be evaluated. The influence of the interventions on cartilage regeneration could not be evaluated and cartilage biopsy could not be performed for ethical reasons. These analyses would have been important to identify the factors associated with pain relief, either from an anti-inflammatory effect or from cartilage tissue regeneration. Physiological assessment of the hyaline cartilage by means of MRI could not be performed due to the absence of specific MRI cartilage evaluation software at the time that this study was conducted. At 12-month follow-up, there were 
no significant changes in radiographic degree of arthritis, but radiographic analyses are not the ideal method to analyse cartilage changes. The small sample size may have led to type II error and different degrees of knee OA, i.e., from early to severe stages were included, which may confound the analyses. The permuted-block randomization resulted in unbalanced baseline group samples.

\section{Conclusion}

An intra-articular injection of bone marrow-derived, cultureexpanded MSCs with or without the addiction of PRP is effective in improving function and decreasing symptoms at 12-month follow-up. Corticosteroid group only show significant improvement in pain and function KOOS subscores at 12-month follow-up. At the 1st month, all the three therapies showed significant improvement in most of KOOS subscores compared to baseline.

Acknowledgements The authors would like to sincerely acknowledge ESHO Empresa de Serviços Hospitalares S.A and Keila Mara Cassiano.

Author contributions RB, RB, MM, SR: conceptualized and designed the work; participated in the patient's follow-up, acquisition, analysis, interpretation of data. RB, RA: drafted the work. VS, RFA, SR, JEM: revising it critically for important intellectual content; analyzed and interpreted the data. RFA, AB: biologists involved in platelet-rich plasma preparation protocol and procedures, and the culture-expanded MSCs procedures. RB: anesthesiologist physician involved in surgical procedures. RLR, JMO: senior biologists responsible for interpretation of the results and translation interpretation to clinical applications. All author approved the final version to be published.

Funding The authors report no financial funding.

\section{Compliance with ethical standards}

Conflict of interest No financial or non-financial competing interest exists for this manuscript. The manuscript was not influenced by personal or financial relationship with other people or organizations.

Ethical approval Comissão Nacional de Ética em Pesquisa (Brazilian National Ethics in Research Committee)-CONEP, under number 14878813.4.0000.5533.

\section{References}

1. Agung M, Ochi M, Yanada S, Adachi N, Izuta Y, Yamasaki T et al (2006) Mobilization of bone marrow-derived mesenchymal stem cells into the injured tissues after intraarticular injection and their contribution to tissue regeneration. Knee Surg Sports Traumatol Arthrosc 14:1307-1314

2. Amable PR, Carias RB, Teixeira MV, da Cruz Pacheco I, do Amaral RJ, Granjeiro JM et al (2013) Platelet-rich plasma preparation for regenerative medicine: optimization and quantification of cytokines and growth factors. Stem Cell Res Ther 4:67

3. Arroll B, Goodyear-Smith F (2004) Corticosteroid injections for osteoarthritis of the knee: meta-analysis. BMJ 328:869

4. Askari A, Naghizadeh MM, Homayounfar R, Shahi A, Afsarian MH, Paknahad A et al (2016) Increased serum levels of IL-17A and IL-23 are associated with decreased vitamin D3 and increased pain in osteoarthritis. PLoS One 11:e0164757

5. Bastos R, Mathias M, Andrade R, Bastos R, Balduino A, Schott $\mathrm{V}$ et al (2018) Intra-articular injections of expanded mesenchymal stem cells with and without addition of platelet-rich plasma are safe and effective for knee osteoarthritis. Knee Surg Sports Traumatol Arthrosc 26:3342-3350

6. Bendinelli P, Matteucci E, Dogliotti G, Corsi MM, Banfi G, Maroni P et al (2010) Molecular basis of anti-inflammatory action of platelet-rich plasma on human chondrocytes: mechanisms of NF-kappaB inhibition via HGF. J Cell Physiol 225:757-766

7. Campbell KA, Saltzman BM, Mascarenhas R, Khair MM, Verma NN, Bach BR Jr et al (2015) Does intra-articular platelet-rich plasma injection provide clinically superior outcomes compared with other therapies in the treatment of knee osteoarthritis? A systematic review of overlapping meta-analyses. Arthroscopy 31:2213-2221

8. Cole BJ, Karas V, Hussey K, Pilz K, Fortier LA (2017) Hyaluronic acid versus platelet-rich plasma: a prospective, double-blind randomized controlled trial comparing clinical outcomes and effects on intra-articular biology for the treatment of knee osteoarthritis. Am J Sports Med 45:339-346

9. Cordeiro-Spinetti E, de Mello W, Trindade LS, Taub DD, Taichman RS, Balduino A (2014) Human bone marrow mesenchymal progenitors: perspectives on an optimized in vitro manipulation. Front Cell Dev Biol 2:7

10. Dai WL, Zhou AG, Zhang H, Zhang J (2017) Efficacy of plateletrich plasma in the treatment of knee osteoarthritis: a meta-analysis of randomized controlled trials. Arthroscopy 33:659-670.e651

11. Devji T, Guyatt GH, Lytvyn L, Brignardello-Petersen R, Foroutan F, Sadeghirad B et al (2017) Application of minimal important differences in degenerative knee disease outcomes: a systematic review and case study to inform BMJ rapid recommendations. BMJ Open 7:e015587

12. Di Martino A, Di Matteo B, Papio T, Tentoni F, Selleri F, Cenacchi A et al (2019) Platelet-rich plasma versus hyaluronic acid injections for the treatment of knee osteoarthritis: results at 5 years of a double-blind, randomized controlled trial. Am J Sports Med 47:347-354

13. Di Y, Han C, Zhao L, Ren Y (2018) Is local platelet-rich plasma injection clinically superior to hyaluronic acid for treatment of knee osteoarthritis? A systematic review of randomized controlled trials. Arthritis Res Ther 20:128

14. Goncalves R, Cabri J, Pinheiro J, Ferreira P, Gil J (2010) Reliability, validity and responsiveness of the Portuguese version of the Knee Injury and Osteoarthritis Outcome Score-physical function short-form (KOOS-PS). Osteoarthr Cartil 18:372-376

15. Ha C-W, Park Y-B, Kim SH, Lee H-J (2019) Intra-articular mesenchymal stem cells in osteoarthritis of the knee: a systematic review of clinical outcomes and evidence of cartilage repair. Arthroscopy 35:277-288.e272

16. Horwitz EM, Le Blanc K, Dominici M, Mueller I, Slaper-Cortenbach I, Marini FC et al (2005) Clarification of the nomenclature for MSC: the international society for cellular therapy position statement. Cytotherapy 7:393-395

17. Huang Y, Liu X, Xu X, Liu J (2019) Intra-articular injections of platelet-rich plasma, hyaluronic acid or corticosteroids for knee osteoarthritis: a prospective randomized controlled study. Orthopade 48:239-247 
18. Hurley ET, Yasui Y, Gianakos AL, Seow D, Shimozono Y, Kerkhoffs $\mathrm{G}$ et al (2018) Limited evidence for adipose-derived stem cell therapy on the treatment of osteoarthritis. Knee Surg Sports Traumatol Arthrosc 26:3499-3507

19. Iannone F, De Bari C, Dell'Accio F, Covelli M, Cantatore FP, Patella V et al (2001) Interleukin-10 and interleukin-10 receptor in human osteoarthritic and healthy chondrocytes. Clin Exp Rheumatol 19:139-145

20. Imamura M, Ezquerro F, Marcon Alfieri F, Vilas Boas L, TozettoMendoza TR, Chen J et al (2015) Serum levels of proinflammatory cytokines in painful knee osteoarthritis and sensitization. Int J Inflamm 2015:329792-329792

21. Jo CH, Lee YG, Shin WH, Kim H, Chai JW, Jeong EC et al (2014) Intra-articular injection of mesenchymal stem cells for the treatment of osteoarthritis of the knee: a proof-of-concept clinical trial. Stem Cells 32:1254-1266

22. Johnstone B, Hering TM, Caplan AI, Goldberg VM, Yoo JU (1998) In vitrochondrogenesis of bone marrow-derived mesenchymal progenitor cells. Exp Cell Res 238:265-272

23. Joshi Jubert N, Rodriguez L, Reverte-Vinaixa MM, Navarro A (2017) Platelet-rich plasma injections for advanced knee osteoarthritis: a prospective, randomized, double-blinded clinical trial. Orthop J Sports Med 5:2325967116689386

24. Jüni P, Hari R, Rutjes AW, Fischer R, Silletta MG, Reichenbach $S$ et al (2015) Intra-articular corticosteroid for knee osteoarthritis. Cochrane Database Syst Rev. https://doi.org/10.1002/14651858. CD005328.pub3

25. Kapoor M, Martel-Pelletier J, Lajeunesse D, Pelletier JP, Fahmi H (2011) Role of proinflammatory cytokines in the pathophysiology of osteoarthritis. Nat Rev Rheumatol 7:33-42

26. Khatab S, van Buul GM, Kops N, Bastiaansen-Jenniskens YM, Bos PK, Verhaar JA et al (2018) Intra-articular injections of platelet-rich plasma releasate reduce pain and synovial inflammation in a mouse model of osteoarthritis. Am J Sports Med 46:977-986

27. Kim M, Rhee J-K, Choi H, Kwon A, Kim J, Lee GD et al (2017) Passage-dependent accumulation of somatic mutations in mesenchymal stromal cells during in vitro culture revealed by whole genome sequencing. Sci Rep 7:14508

28. Kim YS, Koh YG (2018) Comparative matched-pair analysis of open-wedge high tibial osteotomy with versus without an injection of adipose-derived mesenchymal stem cells for varus knee osteoarthritis: clinical and second-look arthroscopic results. Am J Sports Med 46:2669-2677

29. Koh Y-G, Choi Y-J (2012) Infrapatellar fat pad-derived mesenchymal stem cell therapy for knee osteoarthritis. Knee 19:902-907

30. Koh Y-G, Kwon O-R, Kim Y-S, Choi Y-J (2014) Comparative outcomes of open-wedge high tibial osteotomy with platelet-rich plasma alone or in combination with mesenchymal stem cell treatment: a prospective study. Arthroscopy 30:1453-1460

31. Lamo-Espinosa JM, Mora G, Blanco JF, Granero-Moltó F, NuñezCórdoba JM, Sánchez-Echenique C et al (2016) Intra-articular injection of two different doses of autologous bone marrow mesenchymal stem cells versus hyaluronic acid in the treatment of knee osteoarthritis: multicenter randomized controlled clinical trial (phase I/II). J Transl Med 14:246

32. Laver L, Marom N, Dnyanesh L, Mei-Dan O, Espregueira-Mendes J, Gobbi A (2017) PRP for degenerative cartilage disease: a systematic review of clinical studies. Cartilage 8:341-364

33. Lin KY, Yang CC, Hsu CJ, Yeh ML, Renn JH (2019) Intraarticular injection of platelet-rich plasma is superior to hyaluronic acid or saline solution in the treatment of mild to moderate knee osteoarthritis: a randomized, double-blind, triple-parallel, placebo-controlled clinical trial. Arthroscopy 35:106-117

34. Liu Y, Peng H, Meng Z, Wei M (2015) Correlation of IL-17 level in synovia and severity of knee osteoarthritis. Med Sci Monit 21:1732-1736

35. Martin CL, Browne JA (2018) Intra-articular corticosteroid injections for symptomatic knee osteoarthritis: what the orthopaedic provider needs to know. J Am Acad Orthop Surg. https://doi. org/10.5435/jaaos-d-18-00106

36. McIntyre JA, Jones IA, Han B, Vangsness CT (2018) Intra-articular mesenchymal stem cell therapy for the human joint: a systematic review. Am J Sports Med 46:3550-3563

37. Meheux CJ, McCulloch PC, Lintner DM, Varner KE, Harris JD (2016) Efficacy of intra-articular platelet-rich plasma injections in knee osteoarthritis: a systematic review. Arthroscopy 32:495-505

38. Ogura T, Ackermann J, Barbieri Mestriner A, Merkely G, Gomoll AH (2018) Minimal clinically important differences and substantial clinical benefit in patient-reported outcome measures after autologous chondrocyte implantation. Cartilage. https://doi. org/10.1177/19476035187998391947603518799839

39. Pas HI, Winters M, Haisma HJ, Koenis MJJ, Tol JL, Moen MH (2017) Stem cell injections in knee osteoarthritis: a systematic review of the literature. Br J Sports Med 51:1125-1133

40. Pers YM, Ruiz M, Noël D, Jorgensen C (2015) Mesenchymal stem cells for the management of inflammation in osteoarthritis: state of the art and perspectives. Osteoarthr Cartil 23:2027-2035

41. Phinney DG, Pittenger MF (2017) Concise review: MSC-derived exosomes for cell-free therapy. Stem Cells 35:851-858

42. Pittenger MF, Mackay AM, Beck SC, Jaiswal RK, Douglas R, Mosca JD et al (1999) Multilineage potential of adult human mesenchymal stem cells. Science 284:143-147

43. Robinson PG, Murray IR, West CC, Goudie EB, Yong LY, White TO et al (2018) Reporting of mesenchymal stem cell preparation protocols and composition: a systematic review of the clinical orthopaedic literature. Am J Sports Med 47:991-1000

44. van Buul GM, Koevoet WLM, Kops N, Bos PK, Verhaar JAN, Weinans $\mathrm{H}$ et al (2011) Platelet-rich plasma releasate inhibits inflammatory processes in osteoarthritic chondrocytes. Am J Sports Med 39:2362-2370

45. Wang Y, Zhang Z, Chi Y, Zhang Q, Xu F, Yang Z et al (2013) Long-term cultured mesenchymal stem cells frequently develop genomic mutations but do not undergo malignant transformation. Cell Death Dis 4:e950

46. Wang Z, Zheng C, Zhong Y, He J, Cao X, Xia H et al (2017) Interleukin-17 can induce osteoarthritis in rabbit knee joints similar to Hulth's method. Biomed Res Int 2017:2091325

47. Wojdasiewicz P, Poniatowski LA, Szukiewicz D (2014) The role of inflammatory and anti-inflammatory cytokines in the pathogenesis of osteoarthritis. Mediat Inflamm 2014:561459

48. Wong KL, Lee KBL, Tai BC, Law P, Lee EH, Hui JHP (2013) Injectable cultured bone marrow-derived mesenchymal stem cells in varus knees with cartilage defects undergoing high tibial osteotomy: a prospective, randomized controlled clinical trial with 2 years' follow-up. Arthroscopy 29:2020-2028

Publisher's Note Springer Nature remains neutral with regard to jurisdictional claims in published maps and institutional affiliations. 


\section{Affiliations}

\section{Ricardo Bastos ${ }^{1,2,3,4,5,15}$. Marcelo Mathias ${ }^{2} \cdot$ Renato Andrade $^{1,3,6} \cdot$ Ronaldo J. F. C. Amaral $^{7,8,9}$. Vinicius Schott ${ }^{2} \cdot$ Alex Balduino $^{10} \cdot$ Raquel Bastos $^{11}$ • J. Miguel Oliveira ${ }^{4,5,12} \cdot$ Rui L. Reis ${ }^{4,5,12} \cdot$ Scott Rodeo $^{13}$. João Espregueira-Mendes ${ }^{1,3,5,14}$ [D}

Ricardo Bastos

rbastos@espregueira.com

Marcelo Mathias

marcelomathias@ymail.com

Renato Andrade

randrade@espregueira.com

Ronaldo J. F. C. Amaral

ronaldoamaral@rcsi.ie

Vinicius Schott

drschott@bol.com.br

Alex Balduino

balduino@uva.br

Raquel Bastos

raquelcbbastos@gmail.com

J. Miguel Oliveira

miguel.oliveira@i3bs.uminho.pt

Rui L. Reis

rgreis@i3bs.uminho.pt

Scott Rodeo

rodeos@hss.edu

1 Clínica do Dragão, Espregueira-Mendes Sports Centre FIFA Medical Centre of Excellence, Porto, Portugal

2 Universidade Federal Fluminense, Niterói, Rio de Janeiro, Brazil

3 Dom Henrique Research Centre, Porto, Portugal
4 3B's Research Group-Biomaterials, Biodegradables and Biomimetics, Headquarters of the European Institute of Excellence on Tissue Engineering and Regenerative Medicine, University of Minho, AvePark, Parque de Ciência e Tecnologia, Zona Industrial da Gandra, Barco, 4805-017 Guimarães, Portugal

5 ICVS/3B's-PT Government Associate Laboratory, Braga/Guimarães, Portugal

6 Faculty of Sports, University of Porto, Porto, Portugal

7 Kearney Lab, Department of Anatomy, Royal College of Surgeons in Ireland (RCSI), Dublin 2, Ireland

8 Tissue Engineering Research Group, Department of Anatomy, RCSI, Dublin 2, Ireland

9 Centre for Research in Medical Devices (CURAM), National University of Ireland Galway, Galway, Ireland

10 Excellion, Nireói, Rio de Janeiro, Brazil

11 Hospital Lusíadas, Porto, Portugal

12 The Discoveries Centre for Regenerative and Precision Medicine, Headquarters at University of Minho, Avepark, Barco, 4805-017 Guimarães, Portugal

13 Sports Medicine and Shoulder Surgery, Hospital for Special Surgery, New York, NY, USA

14 Orthopaedics Department of Minho University, Braga, Portugal

15 The Biomechanics Group, Department of Mechanical Engineering, Imperial College London, London, England 